\title{
Meronlike Spin Textures in In-Plane-Magnetized Thin Films
}

\author{
Jaianth Vijayakumar®, ${ }^{1}$ Yu Li $\odot,{ }^{2}$ David Bracher, ${ }^{1}$ Craig W. Barton $\odot,{ }^{2}$ Michael Horisberger, ${ }^{3}$ \\ Thomas Thomson $\odot,{ }^{2}$ Jim Miles $\odot,{ }^{2}$ Christoforos Moutafis, ${ }^{2}$ Frithjof Nolting $\odot,{ }^{1}$ and C.A.F. Vaz $\odot^{1, *}$ \\ ${ }^{1}$ Swiss Light Source, Paul Scherrer Institut, Villigen PSI 5232, Switzerland \\ ${ }^{2}$ Nano Engineering and Spintronic Technologies (NEST), Department of Computer Science, University of \\ Manchester, Oxford Road, Manchester M13 9PL, United Kingdom \\ ${ }^{3}$ Laboratory of Neutron and Muon Instrumentation, Paul Scherrer Institut, Villigen PSI 5232, Switzerland
}

(Received 16 May 2020; revised 11 August 2020; accepted 15 October 2020; published 13 November 2020)

\begin{abstract}
The interfacing of magnetic materials with nonmagnetic heavy metals with a large spin-orbit coupling, such as Pt, results in an asymmetric exchange interaction at the interface due to the Dzyaloshinskii-Moriya interaction (DMI), which in turn leads to the formation of skyrmions and topological spin structures in perpendicularly magnetized multilayers. Here, we show that out-of-plane spin domains with lateral dimensions from $200 \mathrm{~nm}$ to $2 \mu \mathrm{m}$ are stabilized in in-plane magnetized $\mathrm{Ta} / \mathrm{Co} / \mathrm{Pt}$ trilayers. We show that these spin textures are largely insensitive to the direction of the in-plane magnetization switched by either magnetic fields or electric fields applied across a $\mathrm{Si}_{3} \mathrm{~N}_{4}$ gate dielectric. The results of micromagnetic simulations indicate that the DMI is required for the stabilization of such out-of-plane domains and that the presence of surface roughness helps to stabilize larger structures, in agreement with experimental results. We identify these spin structures as meronlike topological textures, characterized by a perpendicular spin texture in an uniformly in-plane magnetized system.
\end{abstract}

DOI: 10.1103/PhysRevApplied.14.054031

\section{INTRODUCTION}

Symmetry breaking at interfaces often leads to the emergence of physical phenomena not present in the bulkcounterpart materials [1,2], an example of which is the onset of a net Dzyaloshinskii-Moriya interaction (DMI) in asymmetric multilayer stacks, where ferromagnetic layers are interfaced with nonmagnetic metals with a large spin-orbit coupling (SOC), such as Pt, Ir, or Pd, that can stabilize skyrmion spin textures in perpendicularly magnetized systems [3-7]. Skyrmions are nontrivial magnetic spin textures considered to offer better functionalities for spintronic and storage devices owing to their topological nature, which provides stability from defects and from external perturbations [8-12]. For example, it has been shown that skyrmions and skyrmionic structures can be displaced using relatively small current densities [12], stabilized under an applied magnetic field [13] and, when interfaced with a gate dielectric, controlled by an applied electric field [14-17]. More generally, the topological nature of a spin texture is characterized by the topological charge or skyrmion winding number [18-21]. A chiral skyrmion is endowed with a topological charge of unity, which is reminiscent of magnetic bubbles at similar length scales with a skyrmion number of unity [13,22]. Besides

*carlos.vaz@psi.ch skyrmionic structures, magnetic vortices, chiral domain walls $[23,24]$, Bloch-point domain walls [25], inhomogeneous cycloidal spin structures [26], and merons [27-29] are also topological in nature and are of great interest as they exhibit a similar topological protection to that of skyrmions, which can be classified into Néel [30-35] and Bloch [8,9,36-40] types according to the type of domain wall present on the structure [24] and are usually found in systems with a strong perpendicular anisotropy. Merons are another type of topological excitation consisting of a winding structure with a topological number of $\pm 1 / 2$ and they resemble a Néel-type skyrmion but in a system with dominant in-plane anisotropy [28]; they have been observed in bulk crystals [41] as well as in thin films of (Co,Zn)Mn [28,42], $\mathrm{NiBr}_{2}[43,44]$, and $\mathrm{GdRu}_{2} \mathrm{Si}_{2}$ [45] and in coupled magnetic multilayer structures [46-49]. In contrast, thin films with in-plane anisotropy have Néel walls [50], which are nonchiral [10], and the question arises as to whether by introducing symmetry-breaking interactions in such a system, topological or chiral magnetic spin textures can be induced. Here, we report the formation of out-of-plane spin structures induced by the DMI in a single trilayer of $\mathrm{Ta} / \mathrm{Co} / \mathrm{Pt}$ exhibiting a dominant in-plane magnetic anisotropy. Further, we observe a magnetoelectric coupling in $\mathrm{Si}_{3} \mathrm{~N}_{4} / \mathrm{Ta} / \mathrm{Co} / \mathrm{Pt}$ using silicon nitride as a gate dielectric [51], manifest through large changes in the magnetic configuration with an applied electric field. 
Our experiments agree with the results of micromagnetic simulations, which confirm the presence of such spin structures in $\mathrm{Ta} / \mathrm{Co} / \mathrm{Pt}$ with an internal spin texture resembling a meron pair. We also show that roughness helps to stabilize larger spin structures.

\section{SAMPLE PREPARATION AND CHARACTERIZATION TECHNIQUES}

In order to introduce a nonzero DMI into our film structure, we fabricate asymmetric chiral magnetic multilayers based on $\mathrm{Ta} / \mathrm{Co} / \mathrm{Pt}$ [52]. Figure 1(a) shows a schematic representation of our final device structure, where a 200nm-thick high-resistivity silicon nitride membrane is used as substrate and as a gate dielectric. Before the metal deposition, the membranes are treated in a piranha solution at $90^{\circ} \mathrm{C}$ (sulfuric acid to hydrogen peroxide 3:1; CAUTION: highly corrosive and dangerous to skin) to remove surface contamination. First, top and bottom electrical contacts consisting of $\mathrm{Cr}(3 \mathrm{~nm}) / \mathrm{Cu}(80 \mathrm{~nm}) / \mathrm{Cr}(3 \mathrm{~nm})$ films are deposited by thermal evaporation and defined using a shadow mask. Next, we perform the magnetic multilayer deposition process using dc-magnetron sputtering. Two sample sets are produced, denoted here as sample sets A and B, respectively. Sample set A (grown at the University of Manchester) is fabricated from elemental targets using a working gas of high purity Ar under a pressure of about $4 \times 10^{-3}$ mbar and a power of a $100 \mathrm{~W}$. The typical base pressure prior to sputtering is approximately $10^{-8}$ mbar. Sample set B (grown at the Paul Scherrer Institute) is deposited in a system with a base pressure of $10^{-7}$ mbar under an Ar flow of $5 \mathrm{sccm}$ (corresponding to a chamber pressure of about $2.5 \times 10^{-3}$ mbar) using a power
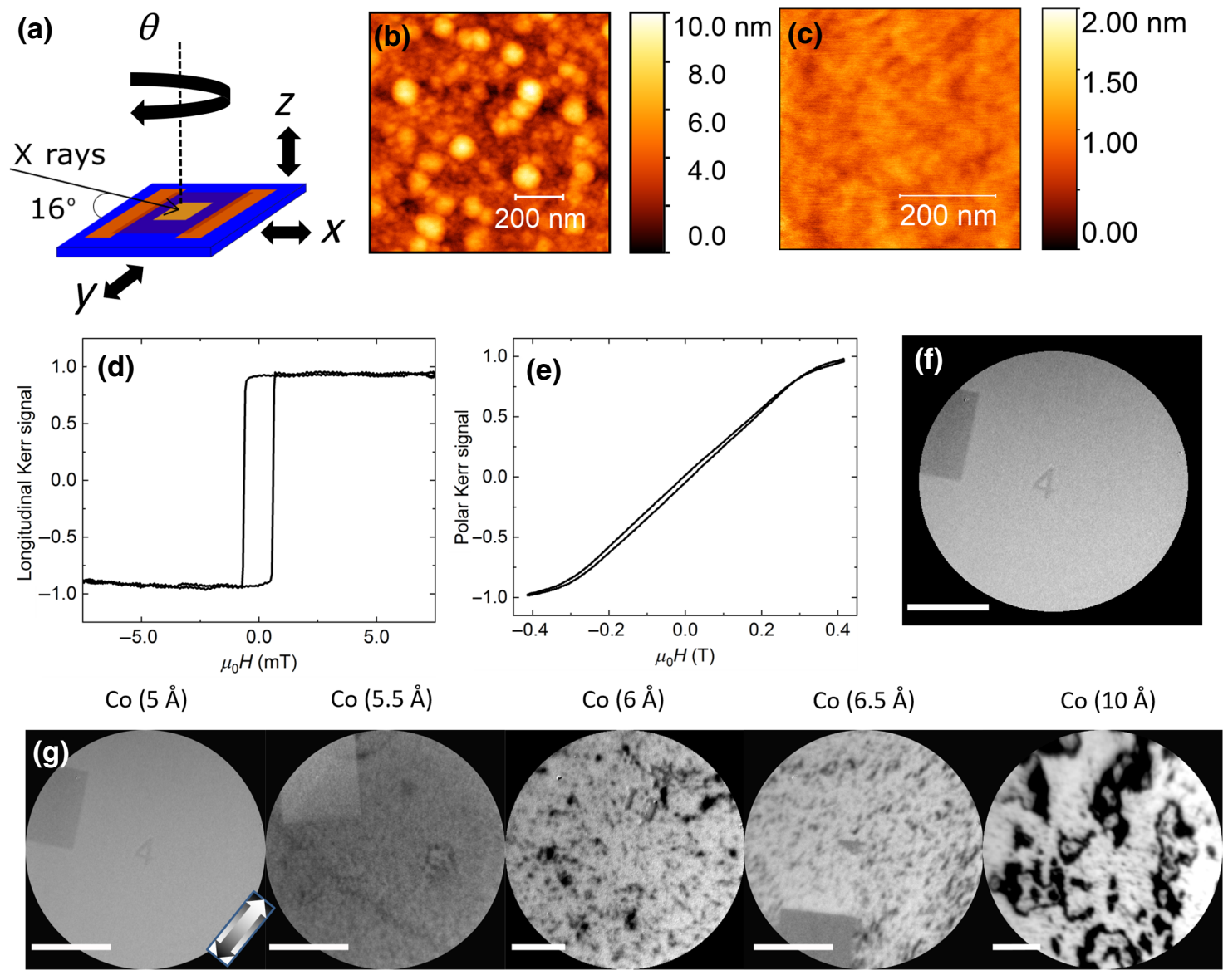

FIG. 1. (a) A schematic of the fabricated sample structure, showing the silicon frame in blue, the membrane in yellow, and the two rectangular top electrodes in orange; the $\mathrm{Ta} / \mathrm{Co} / \mathrm{Pt}$ film is deposited on top of the membrane and overlapping the electrodes. The angle $\theta$ represents the azimuthal rotation of the sample in the microscope. Atomic force microscopy images of (b) $\mathrm{Si}_{3} \mathrm{~N}_{4}$ and (c) $\mathrm{SiO}_{x}$ substrates. (d),(e) Longitudinal and polar MOKE characterization of Ta/Co(6.5 ^)/Pt on $\mathrm{Si}_{3} \mathrm{~N}_{4}$, respectively. (f) An XPEEM Co elemental-contrast image of $\mathrm{Ta} / \mathrm{Co}(5.5 \AA) / \mathrm{Pt}$, showing a uniform Co distribution and the $\mathrm{Cr}$ marker structures (stripe and label " 4 " in the center). (g) The domain configuration of $\mathrm{Si}_{3} \mathrm{~N}_{4} / \mathrm{Ta} / \mathrm{Co} / \mathrm{Pt}$ for different Co layer thicknesses, as denoted in the individual images. The length of the scale bars is $10 \mu \mathrm{m}$; the direction of the magnetic contrast and the XMCD amplitude range (set to \pm 0.03 for 5-6.5 $\AA \mathrm{Co}$ and to \pm 0.06 for $10 \AA \mathrm{Co}$ ) is indicated by the arrow. 
of $200 \mathrm{~W}$ for Ta and $50 \mathrm{~W}$ for Pt and Co. In both sample sets, the magnetic layer thickness is varied to tune the magnetic parameters of the sample and is found to yield consistent results. Sample set A consists of $\mathrm{Ta}(10 \AA) / \mathrm{Co}(6$ $\AA) / \operatorname{Pt}(10 \AA)$ and $\mathrm{Ta}(10 \AA) / \mathrm{Co}(10 \AA) / \operatorname{Pt}(10 \AA)$. Sample set B consists of $\mathrm{Ta}(10 \AA) / \operatorname{Co}(5-10 \AA) / \operatorname{Pt}(15 \AA)$. The two sample sets are deposited on both $\mathrm{Si}_{3} \mathrm{~N}_{4}$ membranes and on reference $\mathrm{SiO}_{x} / \mathrm{Si}(001)$ wafers for subsequent atomic force microscopy (AFM) and magnetic characterization. Although the magnetic films are grown simultaneously, the two types of substrates have significantly different surface morphologies, with rms surface roughness $\sigma=1.3$ $\mathrm{nm}$ and below $0.5 \mathrm{~nm}$ for the $\mathrm{Si}_{3} \mathrm{~N}_{4}$ and $\mathrm{SiO}_{x}$ substrates, respectively, as shown by AFM [see Figs. 1(b) and 1(c)]. For the $\mathrm{Si}_{3} \mathrm{~N}_{4}$ case, the surface lateral-correlation length is estimated as $\xi=46 \mathrm{~nm}$, giving an average radius of curvature (approximately $\xi^{2} / 16 \sigma$ ) of $100 \mathrm{~nm}$. Importantly, the Ta layer interfacing the silicon nitride membrane is kept at $1 \mathrm{~nm}$ to maximize the magnetoelectric coupling effect and also acts to protect the Co from surface adsorbates [51]. Finally, reference markers made of $\mathrm{Cr}$ are patterned by electron-beam lithography used in x-ray photoemission electron microscopy (XPEEM) for sample and image alignment.

The samples are characterized using the magneto-optic Kerr effect (MOKE), superconducting quantum interference device (SQUID) magnetometry, and x-ray photoemission electron microscopy at room temperature. The XPEEM characterization is performed at the SIM beamline [53] (Swiss Light Source, Paul Scherrer Institut), under ultrahigh vacuum (base pressure of $5 \times 10^{-10} \mathrm{mbar}$ ). From XPEEM, one can obtain spatially resolved absorption spectra, while magnetic contrast images are obtained by using the x-ray magnetic circular dichroism (XMCD) effect, which refers to the dependence of light absorption on the magnetization direction, $I \propto \mathbf{M} \cdot \boldsymbol{\gamma}$, where $\mathbf{M}$ is the magnetization vector and $\boldsymbol{\gamma}$ is the x-ray propagation vector, with $\mathrm{XMCD}=\left(I^{+}-I^{-}\right) /\left(I^{+}+I^{-}\right)$(dimensionless), where $I^{ \pm}$is the absorption amplitude for right- and leftpolarized light [54]. The low angle of incidence of the light in our setup, $16^{\circ}$, makes it more sensitive to the inplane magnetization component; it also follows that, upon rotation of the sample with respect to the surface normal [see Fig. 1(a)], the magnetic contrast of the in-plane magnetization changes, while that of the out-of-plane magnetization remains constant [55]. The XMCD images are obtained by acquiring images with circularly right- and left-polarized photons with the energy set to the absorption edge (in this case, Co $L_{3}$ edge) and dividing pixelwise the difference by the sum of the images $[56,57]$. For good statistics, the total integration times for the XMCD images shown here are in the range from 12 to $16 \mathrm{~min}$. Suitable XPEEM sample holders equipped with an electromagnet or equipped with electrical contacts to the sample are used for the application of magnetic and electric fields in situ, respectively [58]. To minimize the risk of electrical discharges and damage to the samples, the XPEEM is operated at a low voltage of $10 \mathrm{kV}$ for the films grown on $\mathrm{Si}_{3} \mathrm{~N}_{4}$ membranes, while for the samples grown on $\mathrm{SiO}_{x}$, we use a standard operation voltage of $15 \mathrm{kV}$. Before XPEEM characterization, the samples are demagnetized using an in-plane alternating magnetic field $(15 \pm 5 \mathrm{mT}$ amplitude).

Micromagnetic simulations are carried out with the MUMAX3 micromagnetic simulation package [59] using the experimental values for the magnetization and outof-plane and in-plane uniaxial magnetic anisotropies, an exchange constant [11] $A=1.0 \times 10^{-11} \mathrm{~J} / \mathrm{m}$, and a DMI value varying from 0 to $2.2 \mathrm{~mJ} / \mathrm{m}^{2}$. The simulation grid chosen is $1786 \times 1786 \times 1$, with a cell size selected for our system of $1.12 \mathrm{~nm} \times 1.12 \mathrm{~nm} \times 0.65 \mathrm{~nm}$, corresponding to a system size of approximately $2000 \mathrm{~nm} \times 2000 \mathrm{~nm} \times$ $0.65 \mathrm{~nm}$. To include the effect of roughness, we use the surface topography of $\mathrm{Si}_{3} \mathrm{~N}_{4}$ measured by AFM, extended to the simulated system size $\left(2000 \times 2000 \mathrm{~nm}^{2}\right)$ by stitching copies of the AFM image. We then calculate the direction of the individual facets from the individual pixels of the AFM image $\left(1.12 \times 1.12 \mathrm{~nm}^{2}\right)$ and assign a corresponding favorable anisotropy direction and DMI vector to the surface plane used for the micromagnetic calculations, i.e., the simulations are carried out on a flat surface but with each pixel having a different favorable easy axis, as illustrated in Fig. 3(a). One consequence is that we neglect the magnetostatic energy contribution from the so called "orange-peel" effect arising from the surface roughness [60,61].

\section{RESULTS AND DISCUSSION}

The magnetic configuration of $\mathrm{Ta} / \mathrm{Co} / \mathrm{Pt}$ as a function of Co thickness (5-10 $\AA$ ) measured with XPEEM under zero applied magnetic field is summarized in Fig. 1(g). The XMCD images show that below $5.5 \AA$, the system is in a uniformly magnetized monodomain state, while above $10 \AA$ we observe large magnetic domains (as expected for such ultrathin films, the magnetic contrast is seen to increase with the Co film thickness). For thicknesses in between, a complex magnetic domain structure is observed, showing the presence of small domains with opposite contrast to that of the larger background domain. MOKE measurements across the sample series indicate that the samples are in-plane magnetized, i.e., we do not find a spin reorientation transition in the $\mathrm{Si}_{3} \mathrm{~N}_{4} / \mathrm{Ta} / \mathrm{Co} / \mathrm{Pt}$ trilayer system. However, the change in domain structure between 6 and $6.5 \AA$ indicates that, at this particular thickness, the magnetic energy balance of the system reaches a saddle point, suggesting a stronger susceptibility to external perturbations. The MOKE results for the $\mathrm{Ta} / \mathrm{Co}(6.5$ $\AA$ A)/Pt sample, presented in Figs. 1(d) and 1(e), display a square easy axis for longitudinal MOKE and a hard-axis 
behavior for polar MOKE, showing that the average magnetization lies in plane. However, the saturation field of $0.4 \mathrm{~T}$ for the out-of-plane direction shows that a significant perpendicular anisotropy is present, which we estimate as $K_{u} \approx H_{\text {sat }} M_{s} / 2-\mu_{0} M_{s}^{2} / 2=-1.1 \mathrm{MJ} / \mathrm{m}^{3}$ using the saturation magnetization value $M_{s}=1.47 \mathrm{MA} / \mathrm{m}$ obtained from SQUID magnetometry (using the nominal Co thickness value). From angular-dependent longitudinal MOKE measurements, we also find the presence of a small inplane uniaxial anisotropy, of the order of $0.25 \mathrm{MJ} / \mathrm{m}^{3}$ (not shown). The presence of a dominant in-plane magnetic anisotropy in our samples is in contrast to other reports in the literature for $\mathrm{Ta} / \mathrm{Co} / \mathrm{Pt}$ multilayer structures, where a spin-reorientation transition is found at around $2 \mathrm{~nm}$ [62-65]. One difference could lie in the fact that most studies employ a Ta buffer layer (2-6 nm thick) to provide a smoother template for the subsequent multilayer growth, also then usually starting with the Pt layer. In the examples where the film growth starts with the Ta layer $[66,67]$, the magnetization is in plane but both the Ta and Co layers used are relatively thick (4-6 nm and 2-2.5 nm, respectively). In our case, we keep the Ta layer deliberately thin $(10 \AA)$; this likely has the effect of keeping the substrate morphology and, through reaction of the Ta with adsorbates from the substrate, of reducing the interface anisotropy of the Co layer. Note that we prepare two film batches from different deposition systems on both $\mathrm{Si}_{3} \mathrm{~N}_{4}$ and $\mathrm{SiO}_{x}$ substrates across a series of thicknesses, showing consistent results, i.e., both XPEEM and magnetometry results show dominant in-plane magnetization, with a strong perpendicular anisotropy $\left(-1.1 \mathrm{MA} / \mathrm{m}^{3}\right)$ that is insufficient to overcome the magnetostatic shape anisotropy $\left(1.3 \mathrm{MA} / \mathrm{m}^{3}\right)$. On the other hand, $\mathrm{Pt} / \mathrm{Co} / \mathrm{Pt}$ films grown under similar conditions exhibit out of plane magnetization [51], indicating that the in-plane magnetic state is related to the $\mathrm{Co} / \mathrm{Ta}$ interface.

To obtain a full mapping of the magnetic configuration, several magnetic contrast images are acquired by rotating the sample with respect to the $\mathrm{x}$-ray propagation direction. The results for the $\mathrm{Ta} / \mathrm{Co}(6.5 \AA) / \mathrm{Pt}$ sample are shown in Fig. 2 for measurements taken at $0^{\circ}, 45^{\circ}$, and $90^{\circ}$. At $0^{\circ}$, the magnetic contrast image indicates the presence of irregularly shaped dark-contrast domains in a brighter background. At $45^{\circ}$, we see the appearance of regions with reverse contrast and at $90^{\circ}$ we find that most contrast in the image has inverted to black. This shows that those regions are in-plane magnetized, with the magnetization pointing predominantly along the $x$ direction of Fig. 1(a) in the $0^{\circ}$ image. In addition, we observe regions with both white and black contrast that remain unchanged with the angle of measurement, indicating regions with out-of-plane magnetization (for example, the regions encircled in Fig. 2); similar results are found for the $\mathrm{SiO}_{x}$ reference sample, although the domain size is on average smaller (not shown). We rule out the

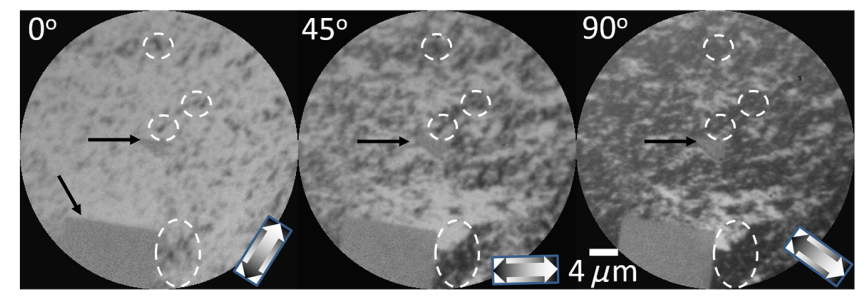

FIG. 2. XMCD images of $\mathrm{Ta} / \mathrm{Co}(6.5 \AA) / \mathrm{Pt}$ on $\mathrm{Si}_{3} \mathrm{~N}_{4}$, acquired at $0^{\circ}, 45^{\circ}$, and $90^{\circ}$ with respect to the $\mathrm{x}$-ray direction. The circles denote out-of-plane magnetized domains. The square at the bottom and the label "4" in the middle of the image are nonmagnetic $\mathrm{Cr}$ markers (indicated by black arrows). The direction of the magnetic contrast is indicated by the bidirectional arrow, with the XMCD amplitude range set to \pm 0.03 .

possibility that these out-of-plane domains correspond to nonhomogeneous regions with locally high perpendicular magnetic anisotropy, since the positions of these domains change after demagnetizing the sample with an in-plane ac magnetic field of about $15 \pm 5 \mathrm{mT}$.

To gain a better understanding of the local spin structure, we carry out micromagnetic simulations; to reproduce the out-of-plane domain structures, we start from an initial configuration where an out-of-plane circular region is surrounded by in-plane spins, as shown in Fig. 3(b), and then we let the system relax to its energy minimum at zero magnetic field for various values of the DMI. The results of the micromagnetic calculations show that when no DMI is present in the system, only the uniform in-plane magnetized state is stable; when the value of the DMI is increased, we find that at a critical value of $1.6 \mathrm{~mJ} / \mathrm{m}^{2}$, a small out-of-plane structure can be stabilized, together with an in-plane antivortex structure [Fig. 3(c)]. The DMI value obtained from the simulation is comparable to the value reported for the $\mathrm{Pt} / \mathrm{Co} / \mathrm{Ta}$ system $[12,52,66]$. Such a winding structure present in a uniformly magnetized background can be seen as a direct consequence of the DMI, which favors a noncollinear configuration at the cost of magnetostatic, exchange, and magnetic anisotropy energy, ultimately determining its lateral size. While the simulated structure for a perfect film is much smaller than the structures that are observed experimentally, when we include the effect of film roughness we find that the size of the inplane and out-of-plane domains increases significantly, as seen in Figs. 3(d) and 3(e), showing that the effect of film roughness is that of stabilizing the out-of-plane domain, partly as a consequence of a reduced magnetostatic energy. This result agrees with the observation of smaller outof-plane structures for $\mathrm{Ta} / \mathrm{Co} / \mathrm{Pt}$ grown on the smoother $\mathrm{SiO}_{x}$ substrates (where we also observe large thermal fluctuations in the domain size). The region with a strong out-of-plane magnetization found in the simulations, indicated by the red color in Fig. 3(e), is relatively wide and is part of an extended in-plane antivortex type spin structure. 

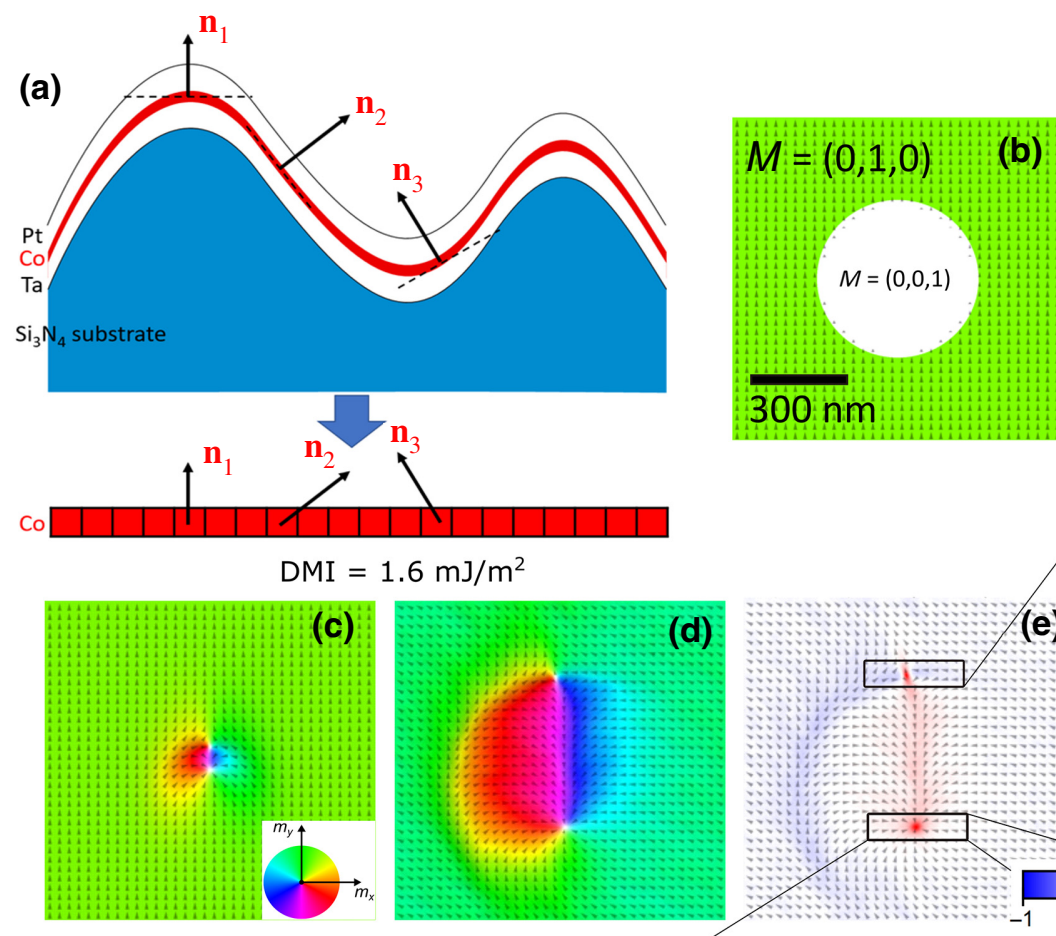

(d)
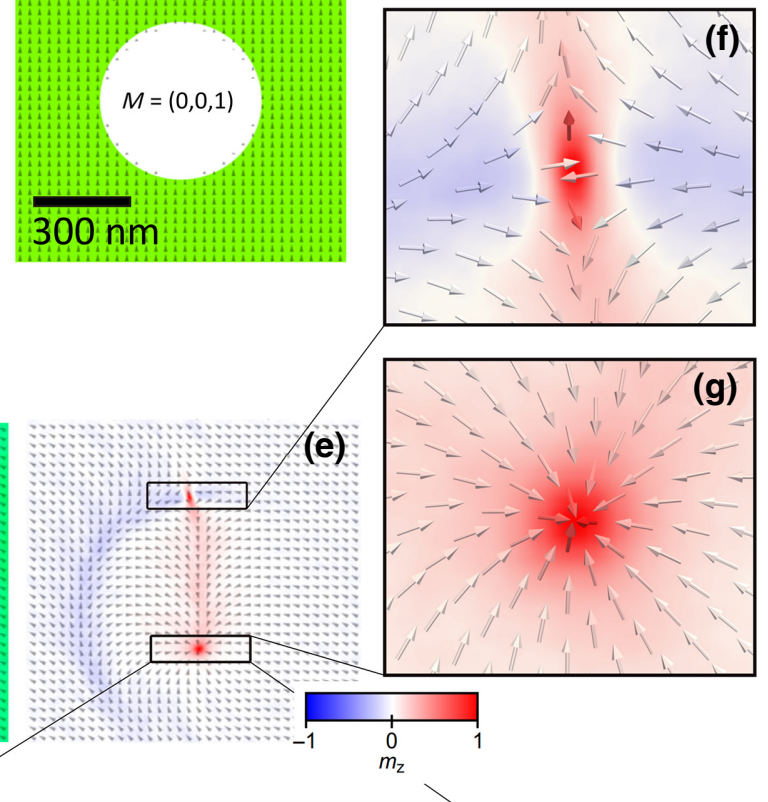

)

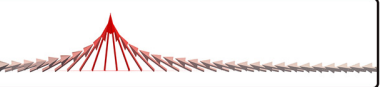

FIG. 3. (a) The roughness profile and anisotropy direction used in the simulation. (b) The initial domain configuration. (c),(d) The final domain configuration for DMI $=1.6 \mathrm{~mJ} / \mathrm{m}^{2}$ (c) without surface roughness and (d) with surface roughness. (e) The vector map of the out-of-plane magnetization component obtained in (d). (f),(g) Enlarged views of individual antimeron and meron structures, respectively. The contour map represents the direction of the in-plane $x$ - and $y$-magnetization-vector components in (b)-(d), while the color scale bar represents the $z$ component of the magnetization vector in (e)-(g).

From the cross section of the spin structure [Fig. 3(e)], we find the topological number to be $\pm 1 / 2$, identifying such structures as a pair of merons. The individual spin structures of the antimeron and meron are shown in Figs. 3(f) and $3(\mathrm{~g})$, respectively: while the meron structure corresponds to a simple antivortex structure, the antimeron spin configuration is more complex, corresponding to two headto-head spin vortex configurations [27-29]. In XPEEM, we observe the entire structure as a single entity with fixed contrast. In XPEEM, the out-of-plane spins should appear with a reduced contrast with the in-plane spins being between gray and white or dark, depending on the contrast direction. However, while we can clearly identify regions with constant contrast for the different measurement angles, the resolution of the data is not sufficient to determine the in-plane spin structure of the meron pairs.

More information about the out-of-plane spin structure can be obtained by following the changes in the domain configuration with an applied magnetic field. We consider first the case of an in-plane static magnetic field, which is applied in steps of about $0.5 \mathrm{mT}$ in plane, along the direction of the light propagation [x in Fig. 1(a)], with the sample then measured at remanence. After applying a magnetic field of $+4 \mathrm{mT}$ [above the coercive field, see Fig. 1(d)], we obtain a magnetic state corresponding to a quasiuniform in-plane magnetized state but with many smaller regions with a reduced contrast level (indicating out-of-plane magnetization) [Fig. 4(a)]; after applying a reverse magnetic field of $-2 \mathrm{mT}$, we find that the magnetic contrast of the in-plane magnetization reverses, while the magnetic contrast of the out-of-plane regions remains largely unchanged [Fig. 4(b)]. An enlarged view of such regions of the sample and the respective XMCD signal profile are shown in Figs. 4(c)-4(e). One finds, in particular, that the contrast of the out-of-plane region remains the same when the neighboring in-plane spins change direction, showing that the out-of-plane spin structures are robust and largely insensitive to in-plane magnetic fields and to the surrounding in-plane magnetization. This process is reproducible and we observe the same effect for most perpendicular spin structures. However, the out-ofplane domains can be modified by larger in-plane and out-of-plane applied magnetic fields. In some cases, we find that the out-of-plane domain can also be completely 


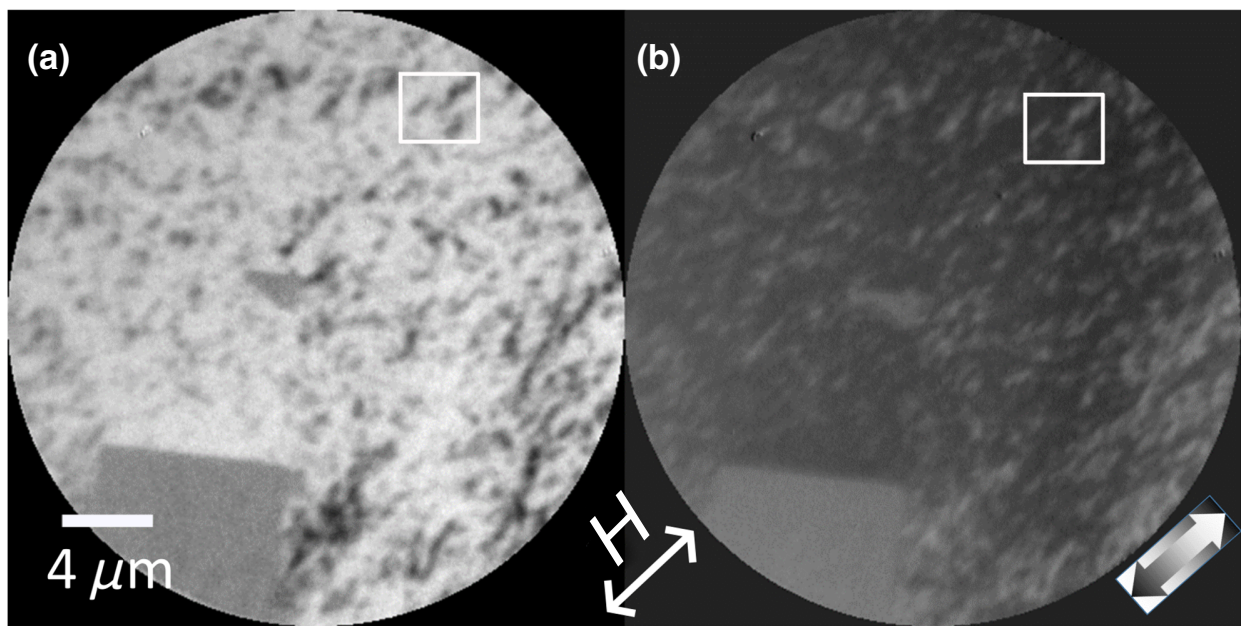

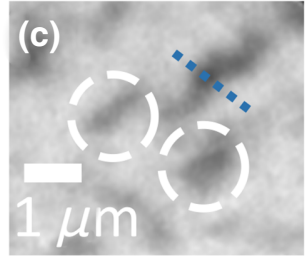

(f)
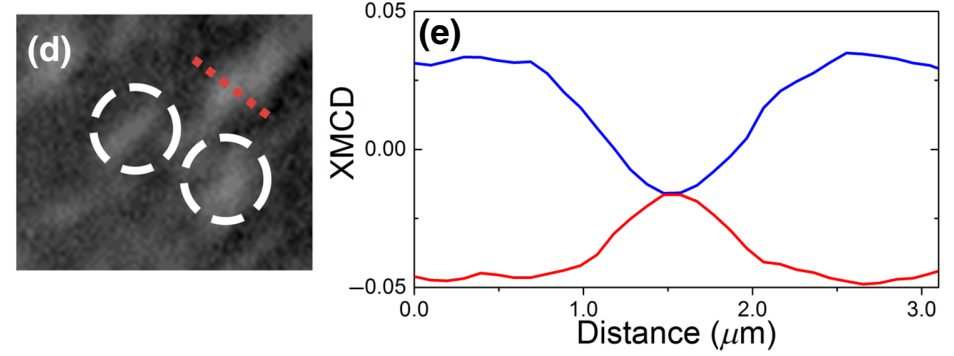

FIG. 4. An overview of the change in the magnetic domain structure after an in-plane magnetic field of (a) $2 \mathrm{mT}$ and (b) $-2 \mathrm{mT}$ is applied to the sample and measured at remanence. (c),(d) Enlarged images of the domain configuration in the white box shown in (a) and (b). The dashed white circles indicate regions with perpendicular spins. The direction of the magnetic contrast is indicated by the arrow (XMCD amplitude range set to \pm 0.06 ). (e) XMCD profiles across the dotted blue and red lines marked in (c) and (d), respectively. (f) Micromagnetic simulations showing the effect of the in-plane magnetic field on the perpendicular spin structure shown in Figs. 3(d) and 3(e), where the contour map represents the in-plane components of the magnetization vector.

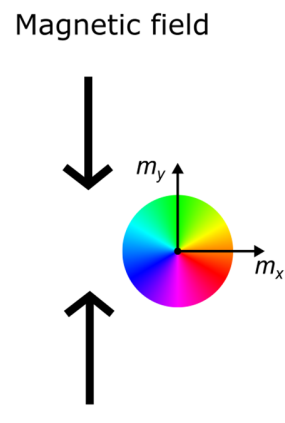

$0 \mathrm{mT}$

$5 \mathrm{mT}$

$10 \mathrm{mT}$

\begin{abstract}
$5 \mathrm{mT}$
\end{abstract}
$0 \mathrm{mT}$

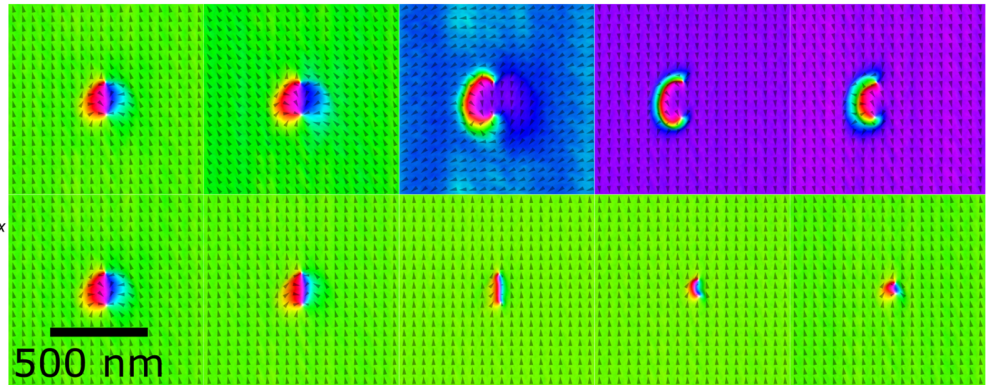

annihilated with a large in-plane magnetic field (from $\pm 2 \mathrm{mT}$ ), which further confirms that these out-of-plane structures are not pinned to a specific region. The results of micromagnetic simulations when an in-plane magnetic field is applied to the spin structure shown in Figs. 3(d) and 3(e) are shown in Fig. 4(f). When the magnetic field is applied along the direction of the in-plane magnetization, we find that its main effect is that of reducing the transverse in-plane components surrounding the meron structure, while the out-of-plane component of the meronpair structure is found to be stable up to relatively high fields. When the magnetic field is applied in the opposite in-plane direction, the transverse spin components of the meron structure rotate around the perpendicular spin component and expand slightly without collapsing, in qualitative agreement with the experimental results.

The effect of applying an out-of-plane magnetic field, in the range of $\pm 3.5 \mathrm{mT}$, is also investigated with XPEEM. In this case, the images are taken while the magnetic field is being applied to the system, with positive fields defined as favoring white magnetic contrast. An overview of the experimental results is shown in Fig. 5(a); the initial state shows the presence of a magnetic texture characterized by small magnetic domains with weak magnetic contrast, corresponding to out-of-plane magnetic domains, in addition to a few reverse (white) in-plane domains (e.g., on the top right). As a negative magnetic field is applied, we observe subtle changes in the magnetic configuration, including a reduction in size of the large in-plane domain (possibly due to a small in-plane magnetic field component) and a reversal in the magnetic contrast of several of the small intermediate light-contrast regions; back at remanence, we find that the light-contrast regions that reversed contrast revert back to the original state. An enlarged view of the changes in the magnetic configuration in the region marked with a white box in Fig. 5(a) is provided in Figs. 5(b)-5(f), showing the magnetic configuration for consecutive values of the applied magnetic field. In particular, one finds 


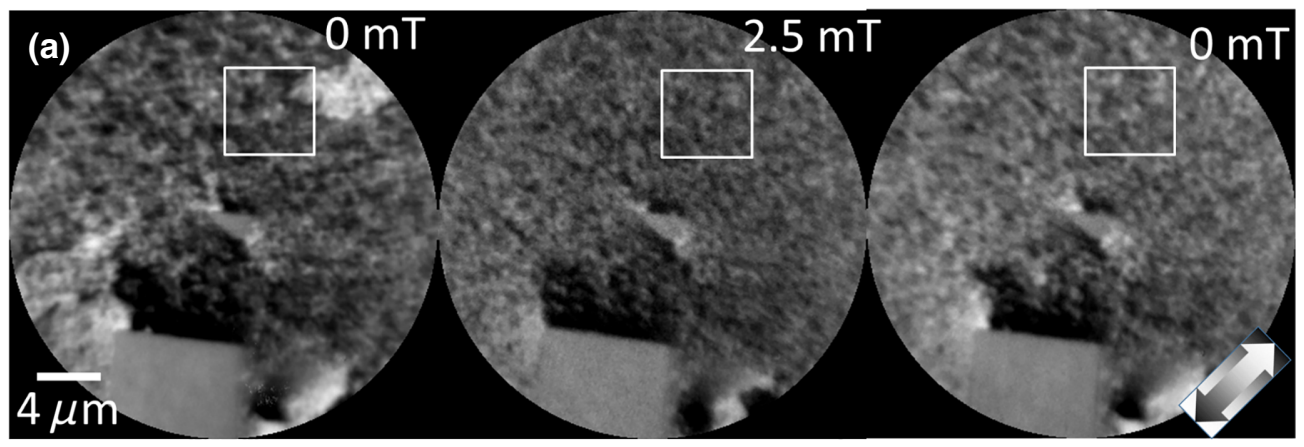

(b)

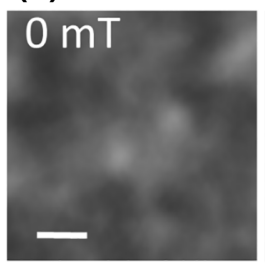

(c)

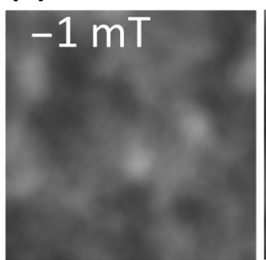

(d)

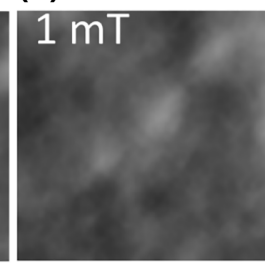

(e)

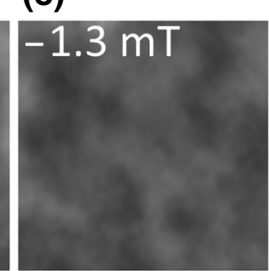

(f)

$1 \mu \mathrm{m}$

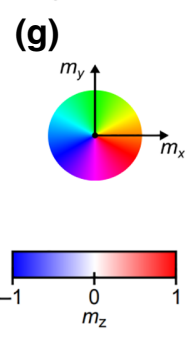

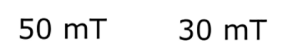

$10 \mathrm{mT}$
$0 \mathrm{mT}$

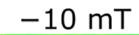

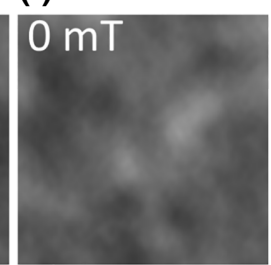

FIG. 5. (a) An overview of the change in the domain structure with an applied outof-plane magnetic field of 0 $\mathrm{mT}, 2.5 \mathrm{mT}$, and $0 \mathrm{mT}$. (b)-(f) Enlarged images of out-ofplane spin structures marked with a white box in (a) as a function of the applied out-of-plane magnetic field. The direction of the magnetic contrast is indicated by the arrow (XMCD amplitude range set to \pm 0.03 ). (g) The effect of the out-of-plane magnetic field on the out-ofplane spin structures determined by micromagnetic simulations. The contour map represents the direction of the $x$ - and $y$-magnetizationvector components for the top row of (g) and the color scale bar represents the direction of the $z$-magnetization component for the bottom row of $(\mathrm{g})$.

that application of a positive magnetic field has little influence on the small circular textures, while a negative field leads first to expansion and then reversal of the magnetic contrast. Back at remanence, the white contrast is recovered, indicating a preferential orientation of the magnetization (although we cannot exclude the presence of a spurious small magnetic biasing field at the sample position). We also image the magnetic structure of the $\mathrm{Ta} / \mathrm{Co} / \mathrm{Pt}$ samples grown on $\mathrm{SiO}_{x}$ under an applied magnetic field, where we find similar effects with an in-plane magnetic field; however, the changes in the out-of-plane spins with a perpendicular magnetic field are small (not shown).

The results of the micromagnetic simulations (showing the in-plane and out-of-plane spin structures) for magnetic fields applied out of plane are shown in Fig. 5(g), where we start with the magnetic configuration shown in the center $(0 \mathrm{mT})$ for both directions of the applied field. We find that while for positive magnetic fields the in-plane spin structure remains largely insensitive to the magnetic field, for negative fields the in-plane spin components exhibit a stronger reduction in size before the magnetization saturates along the field direction (in the simulations, we find the switching fields to be of about $+60 \mathrm{mT}$ and -80 $\mathrm{mT}$ ). In contrast, the out-of-plane spin component is little affected by the magnetic field at low fields, flipping toward the field direction only at large magnetic fields. These results are in qualitative agreement with the experiment, although changes in the spin state tend to occur at much higher fields in the simulations (which do not take into account the effect of thermal excitations).

Finally, we consider the effect of applying an electric field across the $\mathrm{Si}_{3} \mathrm{~N}_{4}$ gate dielectric, motivated by our previous observation of a modulation in the magnetic anisotropy of $\mathrm{Si}_{3} \mathrm{~N}_{4}$-gated $\mathrm{Pt} / \mathrm{Co} / \mathrm{Pt}$ heterostructures [51]. The measurement configuration is shown in Fig. 1(a). The applied voltage is in the range of $\pm 15 \mathrm{~V}(E= \pm 75$ $\mathrm{MV} / \mathrm{m}$ ), with a current limit of $100 \mu \mathrm{A}$ to avoid heating effects. An XMCD image sequence for applied voltages between $+10 \mathrm{~V}$ and $-10 \mathrm{~V}$ is shown in Figs. 6(a)-6(f) for a $\mathrm{Ta} / \operatorname{Co}(6 \AA) / \mathrm{Pt}$ sample. The initial state of the sample before applying electric fields shows a near-uniform magnetization state decorated by gray-contrast domains that are identified as out-of-plane regions. Starting from such a near-uniform in-plane magnetized state at $0 \mathrm{~V}$, we observe the nucleation of a large reverse in-plane magnetic domain with a reverse orientation at $+10 \mathrm{~V}$. The nucleation of the reverse in-plane domain occurs only when positive voltages are applied, by which we deplete electrons at the $\mathrm{Ta} / \mathrm{Si}_{3} \mathrm{~N}_{4}$ interface. The domain wall moves by slow creep, such that sudden jumps of the domain-wall position 

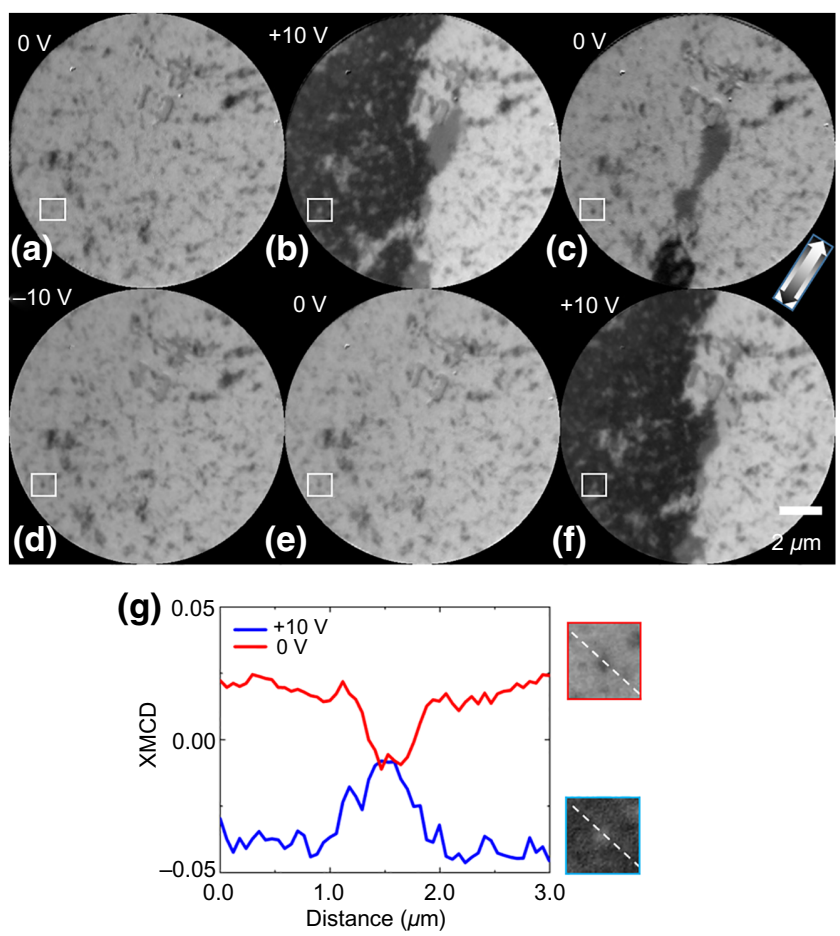

FIG. 6. (a)-(f) The effect of the electric field on the magnetic domain configuration for the $\mathrm{Si}_{3} \mathrm{~N}_{4} / \mathrm{Ta} / \mathrm{Co}(6 \AA) / \mathrm{Pt}$ sample. The area marked by a white square indicates an area with an out-ofplane magnetized region that remains unaffected by the reversal of the surrounding in-plane magnetization. The direction of the magnetic contrast is indicated by the arrow, with the XMCD amplitude range set to \pm 0.07 . (g) The XMCD line profile of the out-of-plane component across the dashed line shown in the insets, corresponding to the structure indicated by a white box in (a) and (b).

occur during the measurement time of $16 \mathrm{~s}$, e.g., the gray area at the center of the image in Fig. 6(b) (this process can be followed in the individual snapshots of $8 \mathrm{~s}$ each). The reverse domain recedes (black to white contrast) upon removal of the applied voltage in a similar slow creep movement and the system returns to its initial configuration. Figure 6(c) shows the state of the system immediately after the removal of the applied $+10 \mathrm{~V}$, where again one captures the movement of the center domain. The domainwall creep suggests the presence of pinning centers for domain-wall displacement, likely caused by local variations in surface morphology and anisotropy. With a negative applied voltage, the domain configuration remains largely unchanged, which we attribute to an increase in the magnetic anisotropy favoring a single domain state, as also suggested by a decrease in the frequency of thermal fluctuations in the XMCD image sequence at negative voltages [51]. During the nucleation of the reverse in-plane domains with the applied voltage, we find that the out-of-plane spins in the regions where nucleation occurred remain largely unchanged; this is shown by the area marked in white in Figs. 6(a)-6(f) and in the corresponding XMCD line profile across the out-of-plane structure for 0 and +10 $\mathrm{V}$, plotted in Fig. 6(g), showing the center region with constant XMCD contrast surrounded by regions with a much larger XMCD value, corresponding to opposite orientations of the in-plane magnetization. In addition, the value of the XMCD contrast in the center area, of about 0.01 , agrees with the expected out-of-plane projection of the moment along the light polarization of approximately $0.05 \sin \left(16^{\circ}\right)$. These results are in agreement with those where the magnetization is reversed by an applied in-plane magnetic field, where the out-of-plane spin structures are also found to be largely insensitive to the surrounding in-plane magnetization.

In the literature, several examples of systems exhibiting the coexistence of in-plane and out-of-plane domains have been reported, achieved by engineering the anisotropy of the system through surface roughness $[61,68]$, local crystallinity [69,70], ion irradiation [71], or in a multilayer stack of in-plane and out-of-plane layers, where the out-ofplane layer can induce an anisotropy in the in-plane layer through the exchange-bias effect $[72,73]$ or by dipolar coupling [10]. The coexistence of in-plane and out-of-plane components (tilted magnetization) has also been observed in a single film at the spin-reorientation transition due to competing in- and out-of-plane anisotropies [74-77] while in the case of $\mathrm{Ta} / \mathrm{Co} / \mathrm{Pt}$ heterostructures with perpendicular magnetic anisotropy, a net DMI leads to the formation of skyrmions with chiral Néel domain walls $[11,52]$. In our case, the presence of out-of-plane domains in an otherwise dominant in-plane anisotropy system is attributed to a strong net DMI that, in combination with a strong outof-plane anisotropy, favors the creation of nonuniform spin structures akin to meron spin textures. Here, we emphasize the observation of meronlike structures at room temperature and without an applied magnetic field in $\mathrm{Ta} / \mathrm{Co} / \mathrm{Pt}$ multilayers, which are known to exhibit skyrmionic states. Our results indicate that by tuning the interfacial properties (such as by modifying the thickness of the metal layers exhibiting the DMI) and the surface roughness, one can induce the formation and stabilization of new topological spin textures.

\section{CONCLUSIONS}

In summary, we observe, using XPEEM, the presence of out-of-plane spin structures in predominantly in-plane magnetized $\mathrm{Ta} / \mathrm{Co} / \mathrm{Pt}$ trilayer structures at a critical thickness of about $6.5 \AA$, at room temperature, and at zero external magnetic field. From micromagnetic simulations, we attribute the formation of such out-of-plane spin structures, which resemble Néel-type skyrmions without a $180^{\circ}$ domain wall and with a topological number of $\pm 1 / 2$, to the DMI. Such spin textures are akin to merons and are characterized by a perpendicular magnetization in an otherwise uniform in-plane magnetization background. 
Surface roughness is found to help stabilize larger structures. From magnetic field and electric field experiments, we find that the meronlike out-of-plane spin structures are largely insensitive to the direction of the surrounding inplane spin direction. Our results suggest the possibility of engineering topologically protected structures in magnetic trilayer films through a suitable control of the interface and morphology, including film roughness, for possible applications in electric-field-controlled memory devices.

\section{ACKNOWLEDGMENTS}

This project is funded by the Swiss National Science Foundation (SNF) (Grant No. 200021-153540). D.B. is funded by the Swiss Nanoscience Institute (SNI) (Grant No. SNI P1502). Y.L. acknowledges the scholarship supported by the China Scholarship Council. We wish to thank Vitaliy Guzenko from the Laboratory of Micro and Nanotechnology for his support with the electron-beam lithography. The magneto-optical Kerr effect measurements using the Durham Magneto Optics NanoMOKE3 ${ }^{\circledR}$ system and the SQUID measurements were carried out at the Laboratory for Mesoscopic Systems, ETH Zurich, Switzerland and the Laboratory for Multiscale Materials Experiments, Paul Scherrer Institut, Switzerland. The AFM measurements were carried out at the Paul Scherrer Institut (PSI) Scanning Probe Microscopy (SPM) User Lab. Part of this work was performed at the Surface/Interface: Microscopy (SIM) beamline of the Swiss Light Source (SLS), PSI, Villigen, Switzerland.

[1] C. A. F. Vaz, F. J. Walker, C. H. Ahn, and S. S. Ismail-Beigi, Intrinsic interfacial phenomena in manganite heterostructures, J. Phys.: Condens. Matter 27, 123001 (2015).

[2] P. Zubko, S. Gariglio, M. Gabay, P. Ghosez, and J.-M. Triscone, Interface physics in complex oxide heterostructures, Ann. Rev. Condens. Matter Phys. 2, 141 (2011).

[3] S. Heinze, K. von Bergmann, M. Menzel, J. Brede, A. Kubetzka, R. Wiesendanger, G. Bihlmayer, and S. Blügel, Spontaneous atomic-scale magnetic skyrmion lattice in two dimensions, Nat. Phys. 7, 713 (2011).

[4] W. Jiang, G. Chen, K. Liu, J. Zang, S. G. E. te Velthuis, and A. Hoffmann, Skyrmions in magnetic multilayers, Phys. Rep. 704, 1 (2017).

[5] A. Soumyanarayanan, M. Raju, A. L. G. Oyarce, A. K. C. Tan, M. Y. Im, A. P. Petrovic, P. Ho, K. H. Khoo, M. Tran, C. K. Gan, F. Ernult, and C. Panagopoulos, Tunable roomtemperature magnetic skyrmions in $\mathrm{Ir} / \mathrm{Fe} / \mathrm{Co} / \mathrm{Pt}$ multilayers, Nat. Mater. 16, 898 (2017).

[6] J. Sampaio, V. Cros, S. Rohart, A. Thiaville, and A. Fert, Nucleation, stability and current-induced motion of isolated magnetic skyrmions in nanostructures, Nat. Nanotechnol. 8, 839 (2013).

[7] A. Hrabec, N. A. Porter, A. Wells, M. J. Benitez, G. Burnell, S. McVitie, D. McGrouther, T. A. Moore, and C. H. Marrows, Measuring and tailoring the Dzyaloshinskii-Moriya interaction in perpendicularly magnetized thin films, Phys. Rev. B 90, 020402(R) (2014).

[8] S. Mühlbauer, B. Binz, F. Jonietz, C. Pfleiderer, A. Rosch, A. Neubauer, R. Georgii, and P. Böni, Skyrmion lattice in a chiral magnet, Science 323, 915 (2009).

[9] X. Z. Yu, Y. Onose, N. Kanazawa, J. H. Park, J. H. Han, Y. Matsui, N. Nagaosa, and Y. Tokura, Real-space observation of a two-dimensional skyrmion crystal, Nature $\mathbf{4 6 5}$, 901 (2010).

[10] G. Chen, S. P. Kang, C. Ophus, A. T. N'Diaye, H. Y. Kwon, R. T. Qiu, C. Won, K. Liu, Y. Wu, and A. K. Schmid, Outof-plane chiral domain wall spin-structures in ultrathin inplane magnets, Nat. Commun. 8, 1 (2017).

[11] C. Moreau-Luchaire, C. Moutafis, N. Reyren, J. Sampaio, C. A. F. Vaz, N. Van Horne, K. Bouzehouane, K. Garcia, C. Deranlot, P. Warnicke, P. Wohlhüter, J. M. George, M. Weigand, J. Raabe, V. Cros, and A. Fert, Additive interfacial chiral interaction in multilayers for stabilization of small individual skyrmions at room temperature, Nat. Nanotechnol. 11, 444 (2016).

[12] F. Hellman, A. Hoffmann, Y. Tserkovnyak, G. S. D. Beach, E. E. Fullerton, C. Leighton, A. H. MacDonald, D. C. Ralph, D. A. Arena, H. A. Dürr, P. Fischer, J. Grollier, J. P. Heremans, T. Jungwirth, A. V. Kimel, B. Koopmans, I. N. Krivorotov, S. J. May, A. K. Petford-Long, J. M. Rondinelli, N. Samarth, I. K. Schuller, A. N. Slavin, M. D. Stiles, O. Tchernyshyov, A. Thiaville, and B. L. Zink, Interface-induced phenomena in magnetism, Rev. Mod. Phys. 89, 1 (2017).

[13] F. Büttner, C. Moutafis, M. Schneider, B. Krüger, C. M. Günther, J. Geilhufe, C. von Korff Schmising, J. Mohanty, B. Pfau, S. Schaffert, A. Bisig, M. Foerster, T. Schulz, C. A. F. Vaz, J. H. Franken, H. J. M. Swagten, M. Kläui, and S. Eisebitt, Dynamics and inertia of skyrmionic spin structures, Nat. Phys. 11, 225 (2015).

[14] T. Srivastava, M. Schott, R. Juge, V. Křižáková, M. Belmeguenai, Y. Roussigné, A. Bernand-Mantel, L. Ranno, S. Pizzini, S.-M. Chérif, A. Stashkevich, S. Auffret, O. Boulle, G. Gaudin, M. Chshiev, C. Baraduc, and H. Béa, Large-voltage tuning of Dzyaloshinskii-Moriya interactions: A route toward dynamic control of skyrmion chirality, Nano Lett. 18, 4871 (2018).

[15] M. Schott, A. Bernand-Mantel, L. Ranno, S. Pizzini, J. Vogel, H. Béa, C. Baraduc, S. Auffret, G. Gaudin, and D. Givord, The skyrmion switch: Turning magnetic skyrmion bubbles on and off with an electric field, Nano Lett. 17, 3006 (2017).

[16] Z. Wang, Z. Ma, and M. J. Grimson, Magnetoelectric coupling and the manipulation of magnetic Bloch skyrmions, Appl. Phys. Lett. 113, 102403 (2018).

[17] W. Zhang, H. Zhong, R. Zang, Y. Zhang, S. Yu, G. Han, G. L. Liu, S. S. Yan, S. Kang, and L. M. Mei, Electrical field enhanced interfacial Dzyaloshinskii-Moriya interaction in $\mathrm{MgO} / \mathrm{Fe} / \mathrm{Pt}$ system, Appl. Phys. Lett. 113, 122406 (2018).

[18] S. Rohart, J. Miltat, and A. Thiaville, Path to collapse for an isolated Néel skyrmion, Phys. Rev. B 93, 214412 (2016).

[19] H.-B. Braun, Topological effects in nanomagnetism: From superparamagnetism to chiral quantum solitons, Adv. Phys. 61, 1 (2012). 
[20] N. Nagaosa and Y. Tokura, Topological properties and dynamics of magnetic skyrmions, Nat. Nanotechnol. 8, 899 (2013).

[21] W. Jiang, X. Zhang, G. Yu, W. Zhang, X. Wang, M. B. Jungfleisch, J. E Pearson, X. Cheng, O. Heinonen, K. L Wang, Y. Zhou, A. Ho, and S. G. E. Velthuis, Direct observation of the skyrmion Hall effect, Nat. Phys. 13, 162 (2017).

[22] C. Moutafis, S. Komineas, C. A. F. Vaz, J. A. C. Bland, T. Shima, T. Seki, and K. Takanashi, Magnetic bubbles in FePt nanodots with perpendicular anisotropy, Phys. Rev. B 76, 104426 (2007).

[23] G. Chen, J. Zhu, A. Quesada, J. Li, A. T. N'Diaye, Y. Huo, T. P. Ma, Y. Chen, H. Y. Kwon, C. Won, Z. Q. Qiu, A. K. Schmid, and Y. Z. Wu, Novel Chiral Magnetic Domain Wall Structure in $\mathrm{Fe} / \mathrm{Ni} / \mathrm{Cu}(001)$ Films, Phys. Rev. Lett. 110, 177204 (2013).

[24] M. J. Benitez, A. Hrabec, A. P. Mihai, T. A. Moore, G. Burnell, D. Mcgrouther, C. H. Marrows, and S. McVitie, Magnetic microscopy and topological stability of homochiral Néel domain walls in a $\mathrm{Pt} / \mathrm{Co} / \mathrm{AlOx}$ trilayer, Nat. Commun. 6, 1 (2015).

[25] S. Da Col, S. Jamet, N. Rougemaille, A. Locatelli, T. O. Mentes, B. Santos Burgos, R. Afid, M. Darques, L. Cagnon, J. C. Toussaint, and O. Fruchart, Observation of Blochpoint domain walls in cylindrical magnetic nanowires, Phys. Rev. B 89, 180405(R) (2014).

[26] S. Meckler, N. Mikuszeit, A. Preßler, E. Y. Vedmedenko, O. Pietzsch, and R. Wiesendanger, Real-Space Observation of a Right-Rotating Inhomogeneous Cycloidal Spin Spiral by Spin-Polarized Scanning Tunneling Microscopy in a Triple Axes Vector Magnet, Phys. Rev. Lett. 103, 157201 (2009).

[27] S.-Z. Lin, A. Saxena, and C. D. Batista, Skyrmion fractionalization and merons in chiral magnets with easy-plane anisotropy, Phys. Rev. B 91, 224407 (2015).

[28] X. Z. Yu, W. Koshibae, Y. Tokunaga, K. Shibata, Y. Taguchi, N. Nagaosa, and Y. Tokura, Transformation between meron and skyrmion topological spin textures in a chiral magnet, Nature 564, 95 (2018).

[29] N. Gao, S. G. Je, M. Y. Im, J. W. Choi, M. Yang, Q. Li, T. Y. Wang, S. Lee, H. S. Han, K. S. Lee, W. Chao, C. Hwang, J. Li, and Z. Q. Qiu, Creation and annihilation of topological meron pairs in in-plane magnetized films, Nat. Commun. 10, 5603 (2019).

[30] I. Kézsmárki, S. Bordács, Peter Milde, E. Neuber, Lukas Eng, Jonathan White, Henrik Ronnow, C. Dewhurst, M. Mochizuki, K. Yanai, H. Nakamura, D. Ehlers, V. Tsurkan, and Alois Loidl, Néel-type skyrmion lattice with confined orientation in the polar magnetic semiconductor $\mathrm{GaV}_{4} \mathrm{~S}_{8}$, Nat. Mater. 14, 1116 (2015).

[31] S. D. Pollard, J. A. Garlow, J. Yu, Z. Wang, Y. Zhu, and H. Yang, Observation of stable Néel skyrmions in cobalt/palladium multilayers with Lorentz transmission electron microscopy, Nat. Commun. 8, 14761 (2017).

[32] O. Boulle, J. Vogel, H. Yang, S. Pizzini, D. De Souza Chaves, A. Locatelli, T. O. Menteş, A. Sala, L. D. BudaPrejbeanu, O. Klein, M. Belmeguenai, Y. Roussigné, A. Stashkevich, S. Mourad Chérif, L. Aballe, M. Foerster, M. Chshiev, S. Auffret, I. M. Miron, and G. Gaudin, Room-temperature chiral magnetic skyrmions in ultrathin magnetic nanostructures, Nat. Nanotechnol. 11, 449 (2016).
[33] F. Tejo, A. Riveros, J. Escrig, K. Y. Guslienko, and O. Chubykalo-Fesenko, Distinct magnetic field dependence of Néel skyrmion sizes in ultrathin nanodots, Sci. Rep. 8, 6280 (2018).

[34] T. Kurumaji, T. Nakajima, V. Ukleev, A. Feoktystov, T. H. Arima, K. Kakurai, and Y. Tokura, Néel-Type Skyrmion Lattice in the Tetragonal Polar Magnet $\mathrm{VOSe}_{2} \mathrm{O}_{5}$, Phys. Rev. Lett. 119, 237201 (2017).

[35] A. K. Srivastava, P. Devi, A. K. Sharma, T. Ma, H. Deniz, H. L. Meyerheim, C. Felser, and S. S. Parkin, Observation of Robust Néel skyrmions in metallic PtMnGa, Adv. Mater. 32, 1904327 (2020).

[36] W. Münzer, A. Neubauer, T. Adams, S. Mühlbauer, C. Franz, F. Jonietz, R. Georgii, P. Böni, B. Pedersen, M. Schmidt, A. Rosch, and C. Pfleiderer, Skyrmion lattice in the doped semiconductor $\mathrm{Fe}_{1-x} \mathrm{Co}_{x} \mathrm{Si}$, Phys. Rev. B 81, 041203(R) (2010).

[37] X. Z. Yu, N. Kanazawa, Y. Onose, K. Kimoto, W. Z. Zhang, S. Ishiwata, Y. Matsui, and Y. Tokura, Near roomtemperature formation of a skyrmion crystal in thin-films of the helimagnet FeGe, Nat. Mater. 10, 106 (2011).

[38] T. Adams, A. Chacon, M. Wagner, A. Bauer, G. Brandl, B. Pedersen, H. Berger, P. Lemmens, and C. Pfleiderer, Long-Wavelength Helimagnetic Order and Skyrmion Lattice Phase in $\mathrm{Cu}_{2} \mathrm{OSeO}_{3}$, Phys. Rev. Lett. 108, 237204 (2012).

[39] A. Tonomura, X. Yu, K. Yanagisawa, T. Matsuda, Y. Onose, N. Kanazawa, H. S. Park, and Y. Tokura, Real-space observation of skyrmion lattice in helimagnet MnSi thin samples, Nano Lett. 12, 1673 (2012).

[40] H. S. Park, X. Yu, S. Aizawa, T. Tanigaki, T. Akashi, Y. Takahashi, T. Matsuda, N. Kanazawa, Y. Onose, D. Shindo, A. Tonomura, and Y. Tokura, Observation of the magnetic flux and three-dimensional structure of skyrmion lattices by electron holography, Nat. Nanotechnol. 9, 337 (2014).

[41] L. Peng, R. Takagi, W. Koshibae, K. Shibata, K. Nakajima, T. H. Arima, N. Nagaosa, S. Seki, X. Yu, and Y. Tokura, Controlled transformation of skyrmions and antiskyrmions in a non-centrosymmetric magnet, Nat. Nanotechnol. 15, 181 (2020).

[42] T. Nagase, M. Komatsu, Y. G. So, T. Ishida, H. Yoshida, Y. Kawaguchi, Y. Tanaka, K. Saitoh, N. Ikarashi, M. Kuwahara, and M. Nagao, Smectic Liquid-Crystalline Structure of Skyrmions in Chiral Magnet $\mathrm{Co}_{8.5} \mathrm{Zn}_{7.5} \mathrm{Mn}_{4}(110)$ Thin Film, Phys. Rev. Lett. 123, 137203 (2019).

[43] B. Göbel, A. Mook, J. Henk, I. Mertig, and O. A. Tretiakov, Magnetic bimerons as skyrmion analogues in in-plane magnets, Phys. Rev. B 99, 060407(R) (2019).

[44] S. K. Kim, Dynamics of bimeron skyrmions in easy-plane magnets induced by a spin supercurrent, Phys. Rev. B 99, 224406 (2019).

[45] N. D. Khanh, T. Nakajima, X. Yu, S. Gao, K. Shibata, M. Hirschberger, Y. Yamasaki, H. Sagayama, H. Nakao, L. Peng, K. Nakajima, R. Takagi, T. Arima, Y. Tokura, and S. Seki, Nanometric square skyrmion lattice in a centrosymmetric tetragonal magnet, Nat. Nanotechnol. 15, 444 (2020).

[46] S. Wintz, C. Bunce, A. Neudert, M. Körner, T. Strache, M. Buhl, A. Erbe, S. Gemming, J. Raabe, C. Quitmann, and J. Fassbender, Topology and Origin of Effective Spin 
Meron Pairs in Ferromagnetic Multilayer Elements, Phys. Rev. Lett. 110, 177201 (2013).

[47] C. Phatak, A. K. Petford-Long, and O. Heinonen, Direct Observation of Unconventional Topological Spin Structure in Coupled Magnetic Discs, Phys. Rev. Lett. 108, 067205 (2012).

[48] T. Shinjo, T. Okuno, R. Hassdorf, ${ }^{\dagger}$ K. Shigeto, and T. Ono, Magnetic vortex core observation in circular dots of permalloy, Science 289, 930 (2000).

[49] F. P. Chmiel, N. W. Price, R. D. Johnson, A. D. Lamirand, J. Schad, G. van der Laan, D. T. Harris, J. Irwin, M. S. Rzchowski, C. B. Eom, and P. G. Radaelli, Observation of magnetic vortex pairs at room temperature in a planar $\alpha-\mathrm{Fe}_{2} \mathrm{O}_{3} / \mathrm{Co}$ heterostructure, Nat. Mater. 17, 581 (2018).

[50] S. Chikazumi, Physics of Ferromagnetism (Wiley, New York, 1997).

[51] J. Vijayakumar, D. Bracher, T. M. Savchenko, M. Horisberger, F. Nolting, and C. A. F. Vaz, Electric field control of magnetism in $\mathrm{Si}_{3} \mathrm{~N}_{4}$ gated $\mathrm{Pt} / \mathrm{Co} / \mathrm{Pt}$ heterostructures, J. Appl. Phys. 125, 114101 (2019).

[52] S. Woo, K. Litzius, B. Krüger, M. Y. Im, L. Caretta, K. Richter, M. Mann, A. Krone, R. M. Reeve, M. Weigand, P. Agrawal, I. Lemesh, M. A. Mawass, P. Fischer, M. Kläui, and G. S. D. Beach, Observation of room-temperature magnetic skyrmions and their current-driven dynamics in ultrathin metallic ferromagnets, Nat. Mater. 15, 501 (2016).

[53] U. Flechsig, F. Nolting, A. Fraile Rodríguez, J. Krempaský, C. Quitmann, T. Schmidt, S. Spielmann, and D. Zimoch, Performance measurements at the SLS SIM beamline, AIP Conf. Proc. 1234, 319 (2010).

[54] J. Stohr and H. C. Siegmann, Magnetism: From Fundamentals to Nanoscale Dynamics (Springer, Berlin, 2006).

[55] L. Le Guyader, A. Kleibert, A. F. Rodríguez, S. El Moussaoui, A. Balan, M. Buzzi, J. Raabe, and F. Nolting, Studying nanomagnets and magnetic heterostructures with $\mathrm{x}$-ray PEEM at the Swiss Light Source, J. Electron Spectrosc. Relat. Phenom. 185, 371 (2012).

[56] J Stohr, Exploring the microscopic origin of magnetic anisotropies with x-ray magnetic circular dichroism (XMCD) spectroscopy, J. Magn. Magn. Mater. 200, 470 (1999).

[57] J. Stohr and S. Anders, X-ray spectro-microscopy of complex materials and surfaces, IBM J. Res. Dev. 44, 535 (2000).

[58] M. Buzzi, C. A. F. Vaz, J. Raabe, and F. Nolting, Electric field stimulation setup for photoemission electron microscopes, Rev. Sci. Instrum. 86, 083702 (2015).

[59] A. Vansteenkiste, J. Leliaert, M. Dvornik, M. Helsen, F. Garcia-Sanchez, and B. Van Waeyenberge, The design and verification of MuMax3, AIP Adv. 4, 107133 (2014).

[60] L. Néel, Sur un nouveau mode de couplage entre les aimantations de deux couches minces ferromagnétiques, C. R. 255, 1545 (1962).

[61] C. A. F. Vaz, S. J. Steinmuller, and J. A. C. Bland, Roughness-induced variation of magnetic anisotropy in ultrathin epitaxial films: The undulating limit, Phys. Rev. B 75, 132402 (2007).

[62] S. Woo, M. Mann, A. J. Tan, L. Caretta, and G. S. D. Beach, Enhanced spin-orbit torques in $\mathrm{Pt} / \mathrm{Co} / \mathrm{Ta}$ heterostructures, Appl. Phys. Lett. 105, 212404 (2014).

[63] M. He, G. Li, Z. Zhu, Y. Zhang, L. Peng, R. Li, J. Li, H. Wei, T. Zhao, X.-G. Zhang, S. Wang, S.-Z. Lin, L. Gu,
G. Yu, J. W. Cai, and B.-G. Shen, Evolution of topological skyrmions across the spin reorientation transition in Pt/Co/Ta multilayers, Phys. Rev. B 97, 174419 (2018).

[64] L. Wang, C. Liu, N. Mehmood, G. Han, Y. Wang, X. Xu, C. Feng, Z. Hou, Y. Peng, X. Gao, and G. Yu, Construction of a room-temperature $\mathrm{Pt} / \mathrm{Co} / \mathrm{Ta}$ multilayer film with ultrahigh-density skyrmions for memory application, ACS Appl. Mater. Interfaces 11, 12098 (2019).

[65] S. Zhang, J. Zhang, Y. Wen, Y. Peng, Z. Qiu, T. Matsumoto, and $\mathrm{X}$. Zhang, Deformation of Néel-type skyrmions revealed by Lorentz transmission electron microscopy, Appl. Phys. Lett. 116, 142402 (2020).

[66] J. Cho, N. H. Kim., S. K. Kang, H. K. Hwang, J. Jung, H. J. Swagten, J. S. Kim, and C. Y. You, The sign of the interfacial Dzyaloshinskii-Moriya interaction in ultrathin amorphous and polycrystalline magnetic films, J. Phys. D: Appl. Phys. 50, 425004 (2017).

[67] A. Moskaltsova, J. Krieft, D. Graulich, T. Matalla-Wagner, and T. Kuschel, Impact of the magnetic proximity effect in $\mathrm{Pt}$ on the total magnetic moment of $\mathrm{Pt} / \mathrm{Co} / \mathrm{Ta}$ trilayers studied by x-ray resonant magnetic reflectivity, AIP Adv. 10, 015154 (2020).

[68] S. J. Steinmuller, C. A. F. Vaz, V. Ström, C. Moutafis, D. H. Y. Tse, C. M. Gürtler, M. Kläui, J. A. C. Bland, and Z. Cui, Effect of substrate roughness on the magnetic properties of thin fcc Co films, Phys. Rev. B 76, 054429 (2007).

[69] S. P. Li, W. S. Lew, J. A. C. Bland, L. Lopez-Diaz, C. A. F. Vaz, M. Natali, and Y. Chen, Magnetic Domain Confinement by Anisotropy Modulation, Phys. Rev. Lett. 88, 087202 (2002).

[70] S. P. Li, W. S. Lew, J. A. C. Bland, L. Lopez-Diaz, M. Natali, C. A. F. Vaz, and Y. Chen, Spin-engineering magnetic media, Nature 415, 600 (2002).

[71] C. Chappert, H. Bernas, J. Ferré, V. Kottler, J.-P. Jamet, Y. Chen, E. Cambril, T. Devolder, F. Rousseaux, V. Mathet, and H. Launois, Planar patterned magnetic media obtained by ion irradiation, Science 280, 1919 (1998).

[72] D. Mitin, A. Kovacs, T. Schrefl, A. Ehresmann, D. Holzinger, and M. Albrecht, Magnetic properties of artificially designed magnetic stray field landscapes in laterally confined exchange-bias layers, Nanotechnology 29, 355708 (2018).

[73] W. Kuch, Xingyu Gao, and J. Kirschner, Competition between in-plane and out-of-plane magnetization in exchange-coupled magnetic films, Phys. Rev. B 65, 064406 (2002).

[74] M. Farle, B. Mirwald-Schulz, A. Anisimov, W. Platow, and K. Baberschke, Higher-order magnetic anisotropies and the nature of the spin-reorientation transition in face-centeredtetragonal $\mathrm{Ni}(001) / \mathrm{Cu}(001)$, Phys. Rev. B 55, 3708 (1997).

[75] S. S. Dhesi, H. A. Dürr, and G. van ver Laan, Canted spin structures in Ni films on stepped Cu(001), Phys. Rev. B 59, 8408 (1999).

[76] C. Klein, R. Ramchal, A. K. Schmid, and M. Farle, Controlling the kinetic order of spin-reorientation transitions in $\mathrm{Ni} / \mathrm{Cu}(100)$ films by tuning the substrate step structure, Phys. Rev. B 75, 193405 (2007).

[77] J. M. Shaw, H. T. Nembach, M. Weiler, T. J. Silva, M. Schoen, J. Z. Sun, and D. C. Worledge, Perpendicular magnetic anisotropy and easy cone state in $\mathrm{Ta} / \mathrm{Co}_{60} \mathrm{Fe}_{20} \mathrm{~B}_{20} / \mathrm{MgO}$, IEEE Magn. Lett. 6, 3500404 (2015). 